\title{
Nonlinear dynamics of periodically focused intense particle beams
}

\author{
R. Pakter ${ }^{1, *}$ and F. B. Rizzato ${ }^{2}$ \\ ${ }^{1}$ Departamento de Matemática, Universidade Luterana do Brasil, Avenida Miguel Tostes 101, 92420, Canoas, RS, Brazil \\ ${ }^{2}$ Instituto de Física, Universidade Federal do Rio Grande do Sul, P. O. Box 15051, 91501-970, Porto Alegre, RS, Brazil
}

(Received 4 January 2002; published 17 May 2002)

\begin{abstract}
We extend a previous study [R. Pakter and F. B. Rizzato, Phys. Rev. Lett. 87, 044801 (2001)] and investigate the nonlinear dynamics of periodically focused intense particle beams. We show that (i) the scenario as the focusing field increases is not the existence of a single threshold above which stable matched (equilibrium) solutions are absent, as believed so far, but the existence of successive regions of stability interrupted by gaps where periodic solutions are either unstable or simply do not exist; (ii) the beam can be focused to tighter radii using stable matched solutions found for focusing field strengths greater than the previous threshold. A comprehensive analysis is carried out as a function of the relevant parameters of the system. Self-consistent simulations validate the findings. The gaps are of crucial importance because they must be avoided if the goal is beam confinement with matched solutions; we develop an analytical model to determine the gap structure, which agrees well with computer simulations.
\end{abstract}

DOI: 10.1103/PhysRevE.65.056503

PACS number(s): 41.85.Ja, 41.75.- $\mathrm{i}$

\section{INTRODUCTION}

The physics of intense beams in periodically focusing systems is an active area of theoretical and experimental research where one looks for external field configurations capable of confining high-current, low-emittance ion or electron beams $[1-5]$. The area is crucial for the development of several advanced particle accelerator applications such as tritium production, spallation neutron sources, heavy ion fusion, coherent radiation sources, and nuclear waste transmutation [6], as well as for applications in basic science. A key aspect of periodically focused beams is their stability properties. Previous studies based on kinetic theory [2] and on the analysis of the beam envelope [7-9] revealed that within a relatively limited range of variation of the focusing field strength, only one equilibrium solution with the beam radius displaying the same periodicity as the external confining field is present; one refers to this solution as the matched solution. Equilibrium in this case results from the balance between defocusing forces associated with electrostatic, thermal, and rigid rotation effects, and focusing forces generated by the confining magnetic field and the self-current effect. The matched solution was shown to present several transient instabilities as the focusing field strength is varied [2], and these instabilities were found to be closely related to nonlinear resonances involving the oscillatory behavior of both the focusing field and the electrostatic perturbations propagating with the beam [9]. In particular, it was shown that above a certain threshold of the focusing field strength the matched solution undergoes a major bifurcation and loses stability $[2,9]$, which creates severe limitations on the practical use of periodic focusing as a confining tool.

In this paper we extend a previous investigation [10], performing an analysis of the nonlinear dynamics exhibited by periodically focused intense particle beams, based on analytical estimates, on the beam envelope equation, and on full

*FAX: +55 51 33167286. Email address: pakter@if.ufrgs.br self-consistent simulations. In particular, we consider a highcurrent beam in a periodic solenoidal focusing field. First of all the analytical model is developed in order to understand the overall nonlinear dynamics of the system. One particular result from the model is the interplay between the various defocusing forces on beam dynamics. It will be seen that relatively dense beams dominated by space-charge effects exhibit nonlinear features, like tangent bifurcations and the corresponding appearance and disappearance of periodic solutions, which are not observed in the absence of spacecharge effects when the beam becomes dominated by thermal and rigid rotation effects, and the dynamics becomes linear. In either of these two cases gaps may be formed as we vary the focusing strength within which no matched solution can be found. This makes the issue of critical relevance if the goal is to operate with stable periodic solutions.

Then the Poincaré mapping technique allied to the Newton-Raphson method [11] is employed to precisely locate and determine the existence and stability of matched solutions in the phase space of the beam envelope model. In particular, we confirm that in space-charge dominated beams the matched solution undergoes a series of direct and inverse bifurcations as the parameters of the system are varied. It is also shown that, although the matched solution analyzed in previous work becomes unstable and eventually vanishes as the focusing field strength increases, stability is recovered for yet larger fields because other stable matched solutions emerge in the phase space. It is thus found that the general scenario as one increases the focusing field is not the existence of a single threshold above which confinement is impossible, but the existence of regions of stability interrupted by gaps where the matched solution either becomes unstable or is completely absent. We investigate the issue and show that the periodicity pattern with which matched solutions can be found is highly sensitive to beam density. The transverse size of the beam is also analyzed, and it is shown that one can effectively focus the beam to tighter radii using the stable matched solutions that are found for focusing strengths greater than the previously established threshold. 
As a final step we supplement the work with a number of self-consistent simulations to validate all the previous findings. Here we see that there is a nice agreement between analytical estimates, simulations involving the envelope equations, and the full self-consistent simulations, a fact that suggests the robustness of our results.

The work is organized as follows. In Sec. II we introduce the model envelope equation and provide initial estimates on its bifurcations and regions of stability and on the interplay between perveance dominated beams (i.e., beams dominated by electrostatic effects $[9,10]$ ) and emittance dominated beams; in Sec. III we perform various simulations with the envelope equation in order to numerically refine the previous analytical estimates; in Sec. IV we run self-consistent beam simulations to check on the overall validity of our lowdimensional model; and in Sec. V we conclude the work.

\section{THE LOW-DIMENSIONAL BEAM ENVELOPE MODEL AND INITIAL ANALYTICAL ESTIMATES}

\section{A. The envelope equation}

In the paraxial approximation the envelope equation for a particle beam in a periodic solenoidal focusing magnetic field reads, in its dimensionless form,

$$
\frac{d^{2} r_{b}}{d s^{2}}+\kappa_{z}(s) r_{b}-\frac{K}{r_{b}}-\frac{1}{r_{b}^{3}}=0 .
$$

In Eq. (1), $s=z / S=\beta_{b} c t / S$ is the dimensionless coordinate along the beam axis, $r_{b}(s)=r_{b, \text { dimensional }} /(S \epsilon)^{1 / 2}$ is the normalized beam envelope radius, and $K=2 q^{2} N_{b} S / \epsilon \gamma_{b}^{3} \beta_{b}^{2} m c^{2}$ is the normalized perveance of the beam, where $S$ is the periodicity length of the magnetic focusing field, $\beta_{b} c$ is the average axial velocity of the beam, $c$ is the speed of light in vacuo, $\epsilon$ is the unnormalized emittance of the beam, $N_{b}$ is the number of particles per unit axial length, and $q, m$, and $\gamma_{b}=\left(1-\beta_{b}^{2}\right)^{-1 / 2}$ are, respectively, the charge, mass, and relativistic factor of the beam particles. The focusing field is characterized by the normalized focusing strength parameter $\kappa_{z}(s)=\kappa_{z}(s+1)=q^{2} B_{z}^{2}(s) S^{2} / 4 \gamma_{b}^{2} \beta_{b}^{2} m^{2} c^{4}$, where $B_{z}(s)$ is the magnetic focusing field on the beam axis. For the sake of simplicity, we consider $\kappa_{z}(s)$ in the form $\kappa_{z}(s)=\sigma_{0}^{2}[1$ $+\delta \cos (2 \pi s)]$, with $\sigma_{0}=\left[\int_{0}^{1} \kappa_{z}(s) d s\right]^{1 / 2}$ representing the vacuum phase advance in the smooth-beam approximation and with $0 \leqslant \delta \leqslant 1$ as the amplitude of the focusing field oscillations. The results to be presented here are independent of the specific form of $\kappa_{z}(s)$. At this point we note that $K$ involves the ratio between two defocusing factors: electrostatic forces due to beam density and thermal forces due to beam emittance. While it becomes suggestive that electrostatic and thermal effects are, respectively, associated with large and small $K$ 's, one purpose of the present paper is to characterize beam dynamics in these two regimes in more detail.

\section{B. Analytical results and estimates}

Let us first make some remarks on the solutions of Eq. (1). We start by noticing that Eq. (1) can be entirely derived from a "time" dependent one-degree-of-freedom Hamiltonian as follows:

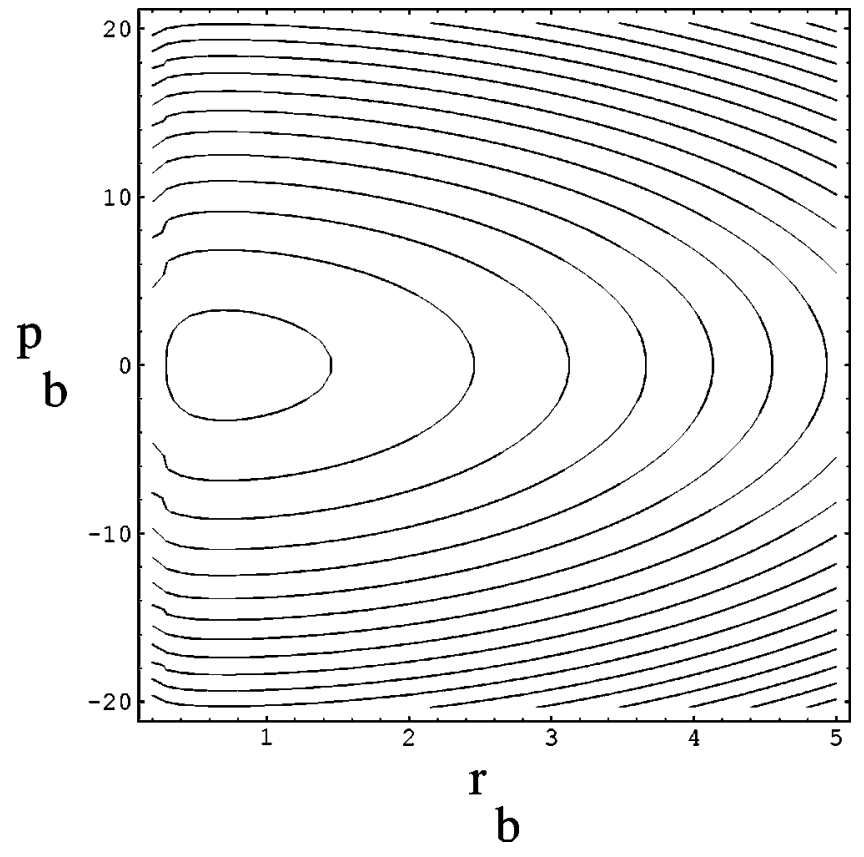

FIG. 1. Contour levels of $H_{0}\left(p_{b}, r_{b}\right)$ for $\sigma_{0}=180^{\circ}$ and $K$ $=3.0$.

$$
H=\frac{p_{b}^{2}}{2}+\kappa_{z}(s) \frac{r_{b}^{2}}{2}-K \ln r_{b}+\frac{1}{2 r_{b}^{2}} \equiv H_{0}+H_{p},
$$

with

$$
\begin{gathered}
H_{0} \equiv \frac{p_{b}^{2}}{2}+\sigma_{0}^{2} \frac{r_{b}^{2}}{2}-K \ln r_{b}+\frac{1}{2 r_{b}^{2}}, \\
H_{p} \equiv \frac{\delta \sigma_{0}^{2}}{2} \cos 2 \pi s r_{b}^{2},
\end{gathered}
$$

and

$$
r_{b}{ }^{\prime}=\frac{\partial H}{\partial p_{b}}=p_{b}, \quad p_{b}{ }^{\prime}=-\frac{\partial H}{\partial r_{b}},
$$

where the primes denote derivatives with respect to $s$.

If the magnetic field is uniform with $\delta=0.0$ and $\kappa_{z}(s)$ $=\sigma_{0}^{2}=$ const, solutions are generated by the autonomous one-degree-of-freedom Hamiltonian $H_{0}$ which is known to be completely integrable yielding regular periodic trajectories only [11]. These integrable trajectories can be seen in a level plot of $H_{0}\left(p_{b}, r_{b}\right)$ as in Fig. 1 . In this case there is one equilibrium radius given by the solution of $\sigma_{0}^{2} r_{b 0}-K / r_{b 0}$ $+1 / r_{b 0}^{3}=0$ in the form

$$
r_{b 0}=\frac{\left[2 K+2\left(K^{2}+4 \sigma_{0}^{2}\right)^{1 / 2}\right]^{1 / 2}}{2 \sigma_{0}} .
$$

The regular periodic trajectories oscillate between maximum and minimum values of $r_{b}, r_{b, \max }$, and $r_{b, \min }$, respectively, from which one can assign a vibrational wave vector mea- 
suring the periodicity of each orbit (recall that as $s$ is a spacelike coordinate we shall use the appropriate "spatial" terminology) in the form $k=k\left(r_{b, \text { max }}\right)$. The equilibrium radius is dynamically stable with the wave number of small linear oscillations of wavelength $\lambda$ around the equilibrium yielded by [9]

$$
\begin{aligned}
k\left(r_{b, \max }\right. & \left.=r_{b 0}\right) \equiv k_{0}=\frac{2 \pi}{\lambda} \\
& =\left[4 \sigma_{0}^{2}+K^{2}-K\left(4 \sigma_{0}^{2}+K^{2}\right)^{1 / 2}\right]^{1 / 2} .
\end{aligned}
$$

Under the condition of a constant $\kappa_{z}=\sigma_{0}^{2}$ one can also determine the wave number of oscillations far away from equilibrium with $r_{b, \max } \rightarrow \infty$, in the form $k\left(r_{b, \max } \rightarrow \infty\right) \equiv k_{\infty}$ $=2 \sigma_{0}$. The wave number tends to this value if for oscillations with large maximal values of $r_{b}$ one discards the terms $1 / r_{b}$ and $1 / r_{b}^{3}$ in Eq. (1) but takes into account the effect of these "centrifugal" forces at $r_{b} \approx 0$; one has essentially a harmonic well blocked at $r_{b}=0$ and thus the factor of 2 in $k_{\infty}$. Let us point out here that $k_{\infty}$ is larger than $k_{0}$. This fact, apparently not properly noticed earlier, has relevant consequences to be discussed next. To analyze beam stability in situations where $\delta \neq 0$ and $\kappa_{z}(s)$ is not constant, we resort to nonlinear dynamics techniques. In the present section we first develop an analytical approach to estimate what will be seen later when we move to the appropriate numerical procedures. Let us then recall from Eq. (2) that

$$
H=H_{0}+H_{p}
$$

$H_{p}$ destroys the integrability of the full Hamiltonian whose nonlinear integrable part is $H_{0}$. Analytical calculations in Hamiltonian systems are more easily done when action-angle variables $(J, \theta)$ for the integrable component are employed. We shall study the issue now.

\section{The integrable part $\mathrm{H}_{0}$}

Our integrable component has a complicated structure as a function of $r_{b}$ and we shall work out an approximate representation for $H_{0}$ in terms of the appropriate action. In our procedure we first determine the rotational wave vector $k(J)$ with which an orbit indexed by an action $J$ oscillates around the equilibrium $r_{b 0}$. Then we will obtain $H_{0}(J)$ as

$$
H_{0}(J)=\int_{J=0}^{J} k\left(J^{\prime}\right) d J^{\prime} .
$$

Our wave vector has the known properties

$$
k(J) \rightarrow\left\{\begin{array}{l}
k_{0} \text { if } r_{b} \rightarrow r_{b 0}(J \rightarrow 0), \\
2 \sigma_{0} \text { if } r_{b} \rightarrow \infty(J \rightarrow \infty),
\end{array}\right.
$$

from which we can interpolate an expression of the form

$$
k(J)=k_{0}+\frac{\Delta_{k} \varepsilon J}{1+\varepsilon J},
$$

with $\Delta_{k} \equiv 2 \sigma_{0}-k_{0}$ and $\varepsilon$ as an yet undetermined quantity measuring the rate at which $k$ increases with the action. From Eqs. (6) and (7) one finds $H_{0}=H_{0}(J)$ in the form

$$
H_{0}(J)=k_{0} J+[\varepsilon J-\ln (1+\varepsilon J)] \frac{\Delta_{k}}{\varepsilon}
$$

and the task now is to evaluate $\varepsilon$. To do that it suffices to compare the first order contributions of an expansion of expression (8) in terms of $J$ with the corresponding expression obtained from a perturbative calculation directly applied to $H_{0}$ of Eq. (2). To expedite the argument, let us sketch what we do here. First we write $r_{b}=r_{b 0}+r$ and expand $H_{0}$, Eq. (2), around the equilibrium at $r_{b}=r_{b 0}$ in powers series of $r$ up to $r^{4}$ :

$$
\begin{aligned}
H_{0} \approx & \frac{p_{b}^{2}}{2}+k_{0}^{2} \frac{r^{2}}{2}-\left(\frac{2}{r_{b 0}^{5}}+\frac{K}{2 r_{b 0}^{3}}\right) r^{3} \\
& +\left(\frac{5}{2 r_{b 0}^{6}}+\frac{K}{4 r_{b 0}^{4}}\right) r^{4},
\end{aligned}
$$

additive constants discarded. Then we introduce new canonical coordinates $(J, \theta)$ according to

$$
\begin{gathered}
r(J, \theta)=\sqrt{\frac{2 J}{k_{0}}} \cos \theta, \\
p_{b}(J, \theta)=-\sqrt{2 k_{0} J} \sin \theta,
\end{gathered}
$$

put these expressions into $H_{0}$, and arrive at

$$
\begin{gathered}
H_{0} \approx k_{0} J+\alpha J^{3 / 2} \sin ^{3} \theta+\beta J^{2} \sin ^{4} \theta, \\
\alpha \equiv-\left(\frac{2}{k_{0}}\right)^{3 / 2}\left(\frac{2}{r_{b 0}^{5}}+\frac{K}{2 r_{b 0}^{3}}\right), \\
\beta \equiv \frac{2}{k_{0}}\left(\frac{5}{2 r_{b 0}^{6}}+\frac{K}{4 r_{b 0}^{4}}\right) .
\end{gathered}
$$

The next step is to find new action-angle variables in order to write $H_{0}$ of Eq. (13) only in terms of the new action. We reserve the calculations for the Appendix and advance the final result:

$$
\begin{aligned}
& H_{0} \approx k_{0} J+\mu J^{2}, \\
& \mu \equiv \frac{3 \beta}{8}-\frac{15 \alpha^{2}}{32 k_{0}} .
\end{aligned}
$$

Now we are in a position to derive an expression for $\varepsilon$. To do this we expand expression (9) up to second order terms in $J$, compare the resulting expression with Eq. (14), and conclude that 


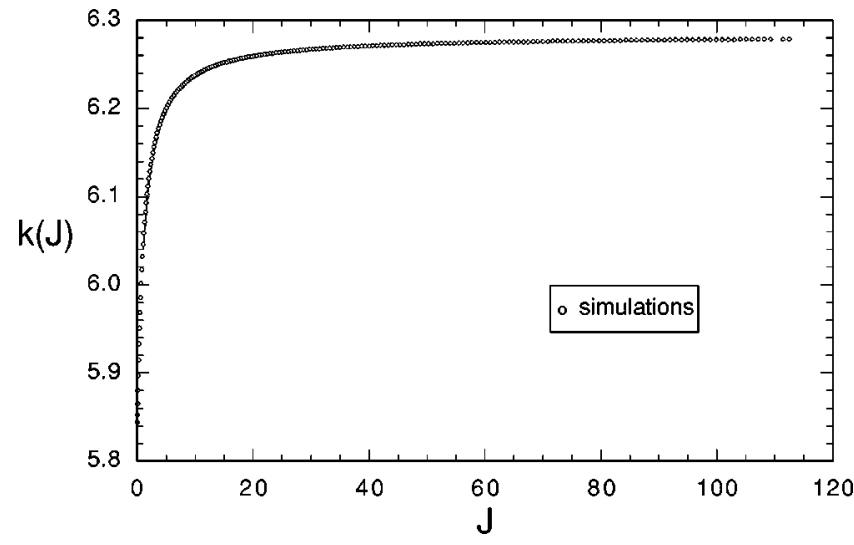

FIG. 2. Analytically and computationally obtained periodicity length $k(J)$ versus $J$ for $K=3.0$ and $\sigma_{0}=180^{\circ}$.

$$
\varepsilon=\frac{\mu}{\Delta_{k}}
$$

Before we proceed we shall anticipate a fraction of the numerical work reserved for the next sections, and test expressions (8) and (9) here. In Fig. 2 we compare the periodicity length of various orbits as obtained directly from the envelope equation (1), with the corresponding result from the analytical approach as represented by expression (8). With $\varepsilon$ determined from Eq. (15), Fig. 2 reveals excellent agreement between numerics and estimates; the simulation results and the analytical curve are almost completely superposed in the figure. It also becomes clear that our wave vector curve increases monotonically up to saturation, which makes reasonable a representation in terms of a single controlling factor like $\varepsilon$. Given the nice accuracy of the analytical approach we now move on to those cases where perturbation is active with $\delta \neq 0$.

\section{Effect of $\boldsymbol{H}_{p}$}

We shall now study the resonant effects produced by the inclusion of $H_{p}$ from expression (2) into the theory. $H_{p}$ has the following structure:

$$
H_{p}=\frac{\delta \sigma_{0}^{2}}{2}\left(r_{b 0}+r\right)^{2} \cos 2 \pi s,
$$

which can be simplified to

$$
H_{p}=\delta \sigma_{0}^{2} r_{b 0} r \cos 2 \pi s+\frac{\delta \sigma_{0}^{2}}{2} r^{2} \cos 2 \pi s
$$

if one drops the purely temporal term. It is apparent that we need the appropriate canonical relation to express $r$ $=r(\theta, J)$. We do not have a formal way to write down the connection so we follow the same informal way leading to the expressions (8), (9), and (15). If $J$ is small one is close to the central fixed point and expressions (11) and (12) can be safely used. When one considers orbits away from the central point some further reasoning must be done. One first recalls that if $J$ is large the orbits are approximately those of a trun- cated oscillator at $r=0$. No restriction is present for $r>0$ and the unperturbed dynamics develops at frequency $2 \sigma_{0}$ in a harmonic way. We collect all the information to write

$$
r(J, \theta) \approx A(J)\left|\cos \frac{\theta}{2}\right| \quad(J \rightarrow \infty)
$$

with $-\pi<\theta \leqslant \pi$. Note that as $\theta$ evolves from $-\pi$ to $\pi$, the argument of the cosine function varies from $-\pi / 2$ to $\pi / 2$, which represents the fact that the oscillator can be seen approximately as blocked at $r=0$. Now let us consider $H_{0}$ when $J$ and $r_{b}$ tend to infinity. From expressions (2) and (9) we write $H_{0} \approx p_{b}^{2} / 2+\sigma_{0}^{2} r_{b}^{2} / 2 \approx 2 \sigma_{0} J$ as $r_{b}$ and $J$ tend to $\infty$, and consider $\theta=0$ where $p_{b}=0$, to finally obtain

$$
A(J)=\sqrt{\frac{8 J}{2 \sigma_{0}}},
$$

which is slightly different from the pure oscillator case, where $A(J)$ would be given by $\sqrt{2 J / 2 \sigma_{0}}$. At this point we shall choose which resonance is to be analyzed. Two of them are of greater relevance for our purposes since they can affect the stability characteristics and even the very existence of the central fixed point: the resonance involving the fundamental harmonic, responsible for the creation of additional matched solutions via tangent bifurcations, and that involving the second harmonic, responsible for period doublings at $r=0$. We shall investigate the resonance at the fundamental harmonic in this work, since, as mentioned, this resonance can provide matched solutions with which one can transport the beam. When $J$ is small one uses expressions (11) and (12) in $H_{p}$ and collects only those harmonic terms with argument $\theta-2 \pi s$ :

$$
H_{p}(J \rightarrow 0) \approx \frac{1}{2} \delta \sigma_{0}^{2} r_{b 0} \sqrt{\frac{2 J}{k_{0}}} \cos (\theta-2 \pi s)
$$

When $J$ is large, we select the more significant $r^{2}$ term and write

$$
\begin{aligned}
H_{p}(J \rightarrow \infty) & \approx \frac{1}{2} \delta \sigma_{0}^{2}\left(\frac{8 J}{2 \sigma_{0}}\right) \cos ^{2}(\theta / 2) \cos (2 \pi s) \\
& =\frac{1}{4} \delta \sigma_{0}^{2}\left(\frac{8 J}{2 \sigma_{0}}\right)(1+\cos \theta) \cos (2 \pi s) \\
& \rightarrow \delta \sigma_{0}^{2}\left(\frac{J}{2 \sigma_{0}}\right) \cos (\theta-2 \pi s)
\end{aligned}
$$

where we dropped off-resonant terms in the last step again. Now we do not know the crossover details from expression (20) to Eq. (21), although the issue lacks importance here. The reason is that for small $J$ the $J^{1 / 2}$ term automatically dominates, while for $J$ large it is the $J^{1}$ term that automatically prevails. In any case we use the additional canonical transformation $\theta-2 \pi s \rightarrow \theta$ and $H-2 \pi J \rightarrow h_{\text {res }}$ to write a final form 


$$
\begin{aligned}
h_{r e s}(J, \theta)= & H_{0}(J)-2 \pi J \\
& +\delta \sigma_{0}^{2}\left[\frac{r_{b 0}}{2} \sqrt{\frac{2 J}{k_{0}}}+f \frac{J}{2 \sigma_{0}}\right] \cos \theta,
\end{aligned}
$$

where we introduce a crossover factor $f$ modeled by $f$ $=J^{\xi} /\left(1+J^{\xi}\right)$, with $\xi=1 / 2$ chosen this way to refine the already nice agreement with simulations. The model focus on resonances of the type $\theta^{\prime} \approx 2 \pi$; resonances of type $\theta^{\prime}$ $\approx 2 n \pi$ with $n$ integer will be analyzed numerically later. Matched solutions are resonances at the fundamental harmonic which manifest themselves as fixed points of the dynamics entailed by $h_{\text {res }}$. Fixed points are defined in the form $\theta^{\prime}=J^{\prime}=0$, and from Eq. (22) along with the proper canonical equations this demands

$$
\begin{gathered}
\theta_{\text {fixed }}^{(+)}=0, \quad \theta_{\text {fixed }}^{(-)}=\pi, \\
g_{ \pm} \equiv \frac{d}{d J} h_{\text {res }}\left(J, \theta_{\text {fixed }}^{( \pm)}\right)=0 .
\end{gathered}
$$

The functions $g_{ \pm}(J)$ are parametrized by control factors like $K, \delta$, and $\sigma_{0}$ and their shape depends on those factors. Take, for instance, $K=0.5, \delta=0.03$, and $\sigma_{0}=182.73^{\circ} \approx 1.015 \pi$ and draw $g_{ \pm}$as in Fig. 3(a). We see that for this particular parametric choice three fixed points coexist since the $g_{+}$ curve intersects the horizontal axis twice while $g$ - intersects the horizontal axis once; changing $\sigma_{0}$ basically shifts the curves vertically, and so we see that fixed points of curve $g_{+}$ appear or disappear in pairs via tangent bifurcations. Next consider a case with much smaller perveance $K=0.01$ and a larger $\delta=0.1$, at $\sigma_{0}=191.16^{\circ}=1.062 \pi$, as pictured in panel Fig. 3(b). Here the curves $g_{+}$and $g_{-}$are both monotonic and a gap is formed which provides a region where no fixed point can be found; otherwise, as $\sigma_{0}$ is varied, one has at most one fixed point at a time. Figure 3(c), which uses $K=3.0, \delta=1.0$, and $\sigma_{0}=180^{\circ}=\pi$, finally represents the intermediary situation where one has a nonmonotonic $g_{+}$ curve along with the presence of the gap; now one has at most the simultaneous presence of two fixed points arising from the solutions of $g_{+}=0$. We thus see that, depending on the parameters, three regimes may be present: nonmonotonicity without gaps, gaps without nonmonotonicity, and nonmonotonicity with gaps. Nonmonotonicity, which is exclusively associated with curve $g_{+}$, can be analyzed along the following lines. $g_{+}$tends to $+\infty$ when $J \rightarrow 0$. Now perveance determines the ascending aspect of the curves and when it is large $g_{+}$also increases at intermediary values of $J$; under this condition, nonmonotonic features are present because one must have a minimum somewhere. On the other hand, when perveance is too small the related ascending behavior and the resulting minimum may not be present. The transition from one regime to the other is estimated when one realizes that in terms of order of magnitude the unperturbed nonlinear frequency increases significantly in that region where $J \sim 1 / \varepsilon$. If at this point the perturbative $\delta / \sqrt{J}$ term in $g_{+}$is already very small, then perveance effects should be noticeable; perveance effects remain unnoticed
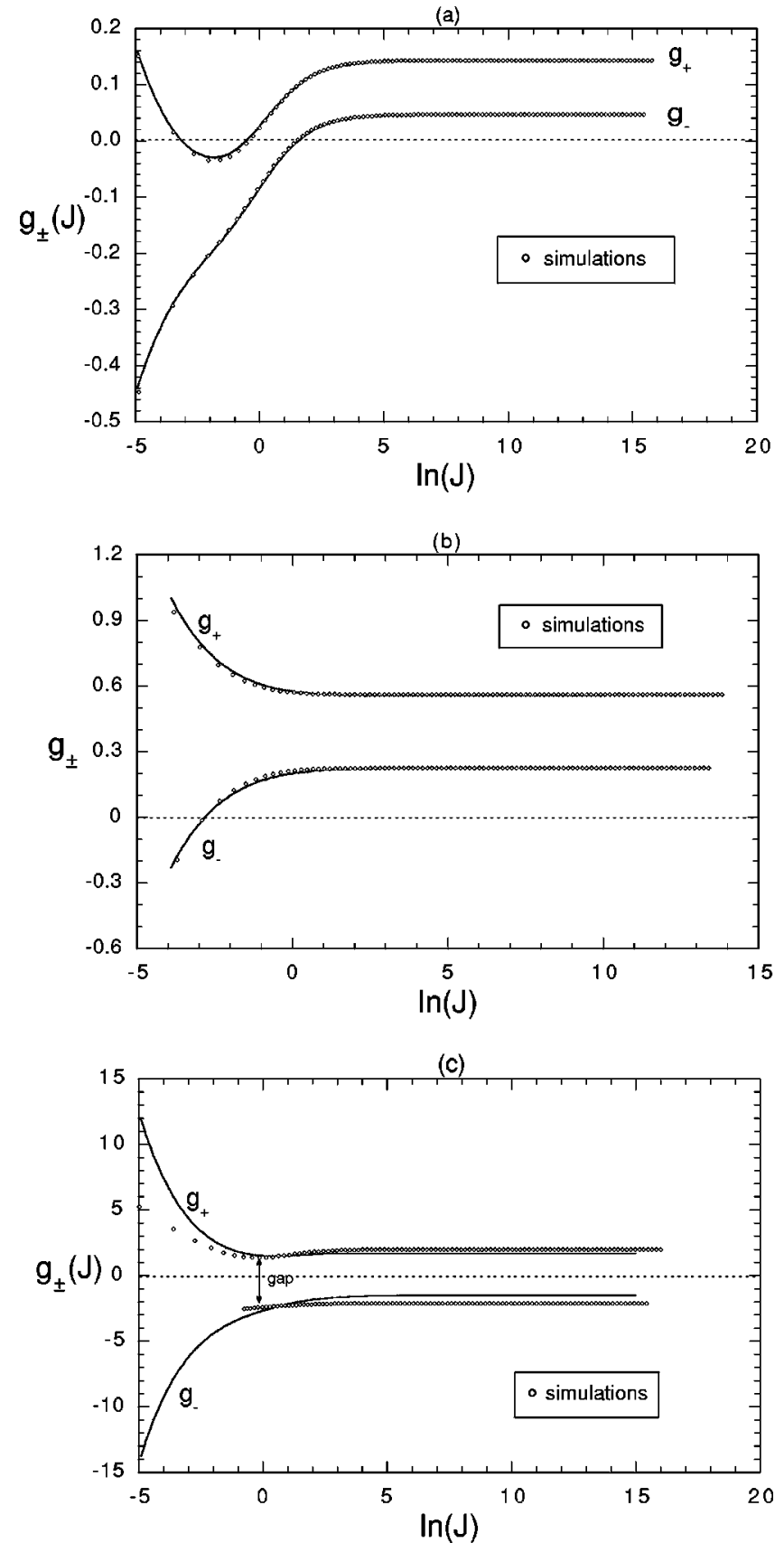

FIG. 3. Curves $g_{+}$and $g_{-}$superimposed on corresponding simulations as discussed in the text. In (a) $K=0.5, \delta=0.03$, and $\sigma_{0}=182.73^{\circ}$; in (b) $K=0.01, \delta=0.1$, and $\sigma_{0}=191.16^{\circ}$; in (c) $K$ $=3.0, \delta=1.0$, and $\sigma_{0}=180^{\circ}$.

otherwise. What is needed to see nonlinearity can be thus translated into the following inequalities coming from $\partial H_{p} / \partial J$, with $H_{p}$ defined in expression (20), and the condition $J \sim 1 / \varepsilon$ :

$$
\frac{\delta \sigma_{0}^{2} r_{b 0}}{\sqrt{k_{0} / \varepsilon}} \ll \Delta_{k} \varepsilon\left(\frac{1}{\varepsilon}\right) \Rightarrow \delta \ll \delta_{c r} \equiv \frac{\Delta_{k}}{\sigma_{0}^{2} r_{b 0}} \sqrt{\frac{k_{0}}{\varepsilon}} .
$$

When $\delta \gg \delta_{c r}$ we expect linear behavior without any tangent bifurcations, and $\delta \sim \delta_{c r}$ defines the marginal regime where 
nonlinearity, if present, should be small. In the linear case of Fig. 3(b), for instance, $\delta=0.1, \quad \delta_{c r} \approx 2.2 \times 10^{-3}$, and $\delta$ $\gg \delta_{c r}$, as it should be here; results based on condition (25) are also consistent in other situations. The other distinctive effects seen in the figures are the gaps, and these get more and more prominent as $\delta$ grows. Gaps are intimately connected with the orbital asymmetry around $r_{b 0}$. If it were not for the asymmetry resulting in expression (18), the linear term in $J$ would be absent from $h_{\text {res }}$ and no gap would be present-one also sees that the gap size grows linearly with $\delta$. Needless to say, the existence of gaps is of foremost importance since they must be identified and avoided if one is interested in the search for matched stable solutions. We finally note that in purely monotonic (or linear) cases the gap is necessarily present.

Our final task here is to compare the theoretical findings with proper simulations of the envelope equations and here we briefly mention more of the heavier numerical work postponed for the next sections. To provide an initial test of our findings we launch several initial conditions at $p_{b}=0$, integrate these conditions forward until their next return to $p_{b}$ $=0$, and compute the associated length interval $\mathcal{S}$. From the integration we obtain the approximate differential frequency $\nu=2 \pi /(2 \mathcal{S})-2 \pi$-which should be compared with curves $g_{ \pm}$- and the approximate action $J=(1 / 2 \pi) \oint p_{b} d r_{b}$ $=(1 / 2 \pi) 2 \int_{0}^{\mathcal{S}} p_{b}^{2} d s$-which are then compared with results obtained from Eq. (24). We superimpose the computed results on the estimates in Fig. 3. It is then seen that our analytical model explains well what is going on in these lowdimensional simulations. The two branches of the simulations arise as a result of initial conditions launched to the left or right hand sides of the equilibrium $\left(r_{b 0}, p_{b}=0\right)$. We point out that, as might be expected, agreement is better for small $\delta$ 's.

\section{SIMULATIONS OF THE ENVELOPE EQUATIONS}

In this section, we analyze the results obtained by direct integration of the beam envelope equation (1). Let us then introduce the numerical tools of analysis in a more formal fashion. We shall look at the phase space and its respective periodic orbits from the optics of Poincare plots and the corresponding stability analysis. In the plots we record the pair $\left(r_{b}, p_{b}\right)$ at integer values of $s[11]$ and evaluate the stability $\alpha$ of periodic orbits which appear as fixed points of the phase space, from $\alpha=\cos \left(k_{f i x}\right) \cdot k_{f i x}$ is the wave number of small linear oscillations around the periodic trajectory, obtained with a Newton-Raphson method. For stable orbits where $k_{f i x}$ is a real number, $|\alpha| \leqslant 1$; if $\alpha$ crosses the upper boundary $\alpha=+1$ the orbit undergoes an inverse tangent bifurcation with a previous unstable fixed point, and if the orbit crosses the lower boundary $\alpha=-1$ it undergoes a period doubling bifurcation, losing stability. According to analytical estimates obtained in the last section, the presence of bifurcations as typical nonlinear features depends on the values of the perveance $K$ and of the perturbing factor $\delta$. We initially address numerically the role of $\delta$. When $\delta=0.0$, one can simply evaluate $\alpha$ for the central fixed point in the form $\alpha$ $=\cos \left(k_{0}\right)$, where $k_{0}$ is defined in Eq. (5). In this case, the
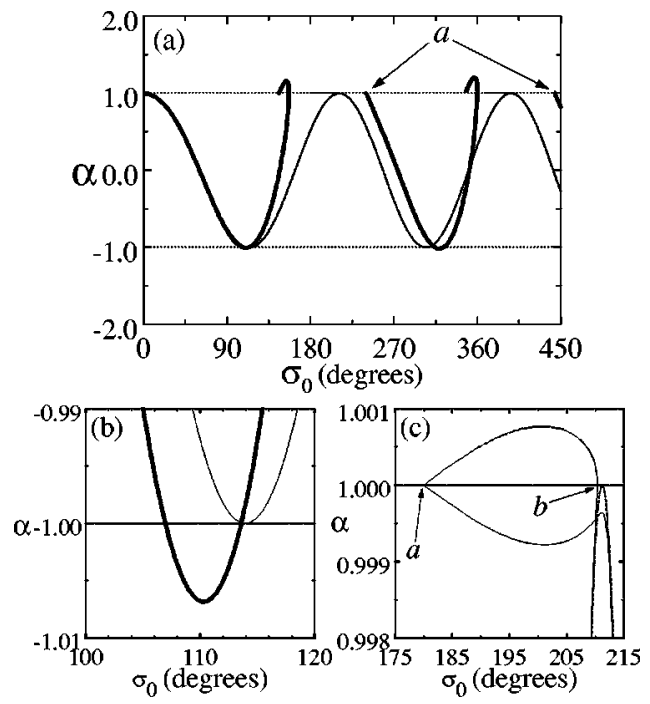

FIG. 4. Stability diagram $\alpha$ vs $\sigma_{0}$ for $K=3.0$ and $\delta=0.0$ (dashed line), $\delta=2.0 \times 10^{-3}$ (thin solid line), and $\delta=1.0$ (thick solid line). In (b) and (c) we expand (a) close to bifurcations.

argument of the cosine function is real, $|\alpha|$ is always bounded by +1 , and no bifurcations occur in the phase space. On the other hand, when the perturbation is turned on with $\delta \neq 0$, various bifurcations may mark their presence on the phase space. These bifurcations are analyzed in Fig. 4, where we plot the stability index $\alpha$ as a function of the vacuum phase advance $\sigma_{0}$ (in degrees) for a uniform magnetic field $\delta=0.0$ (dashed line), a perturbative case with $\delta$ $=2.0 \times 10^{-3} \ll 1$ (thin solid line), and a nonperturbative case with $\delta=1.0$ (thick solid line). Note that the $\delta=0.0$ curve is generally not visible because the $\delta=2.0 \times 10^{-3}$ curve is essentially on top of it for most of the values of $\sigma_{0}$; the only regions where these two curves can be distinguished are close to the bifurcations, as discussed next.

\section{A. Period doubling: The strong $\sigma_{0} \approx 90^{\circ}$ instability}

First of all we observe that when $\delta \neq 0$ the first valley of the curve $\alpha$ vs $\sigma_{0}$ drops to a position slightly below the lower boundary $\alpha=-1$. This is shown in detail in Fig. 4(b), where we see that the $\delta=1.0$ matched solution clearly crosses the $\alpha=-1$ line. This means that at this point the matched solution undergoes a period doubling and becomes unstable, as noticed in a series of previous works in connection with the strong $\sigma_{0}=90^{\circ}$ instability; search for stable solutions traditionally does not go beyond this point. However, if one continues to increase $\sigma_{0}$, we see that the matched solution crosses back over the $\alpha=-1$ horizontal line, recovering stability. The gap of instability for the $\delta=1.0$ matched solution can be estimated from Fig. 4 (b) as $\Delta \sigma_{0} \approx 8^{\circ}$. Although not clear from Fig. 4(a), the $\delta=2.0 \times 10^{-3}$ matched solution also undergoes the same sequence of direct followed by inverse period doublings, the difference being the size of the instability gap, which is much shorter: $\Delta \sigma_{0} \approx 0.016^{\circ}$. In fact, any $\delta \neq 0$ would induce the sequence of bifurcations, with smaller $\delta$ 's, generating linearly shorter instability gaps. The sequence is also displayed in the form of Poincare plots 

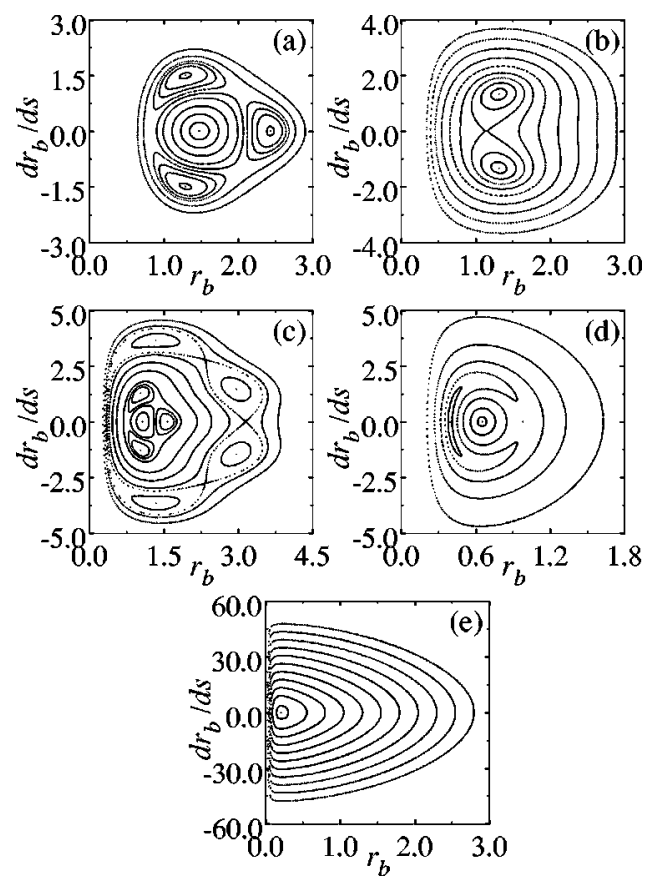

FIG. 5. Poincare plots of the $r_{b}$ vs $d r_{b} / d s$ phase space. $K$ $=3.0$ and (a) $\delta=1.0, \sigma_{0}=75.4^{\circ}$; (b) $\delta=1.0, \sigma_{0}=109^{\circ}$; (c) $\delta$ $=1.0, \sigma_{0}=135^{\circ} ;$ (d) $\delta=2.0 \times 10^{-3}, \sigma_{0}=207^{\circ}$; and (e) $\delta=1.0$, $\sigma_{0}=292^{\circ}$.

in Figs. 5(a) (before period doubling), 5(b) (after period doubling), and 5(c) (after inverse period doubling) for the case $\delta=1.0$.

\section{B. Beyond period doubling}

To analyze the remaining bifurcations that take place in the phase space, one can make use of the analytical estimates derived earlier in Sec. II for guidance. As before, we focus attention on resonances with the same periodicity of the driver since those can establish additional matched solutions. Resonances exist where $g_{ \pm}(J)=0$. If $\delta$ is small one falls into the generic case illustrated by Fig. 3(a). Resonance appears in the phase space when $\sigma_{0} \approx 180^{\circ}$, at $J \rightarrow \infty$, with the unstable fixed point at $\theta=0$ and the stable point at $\theta=\pi$. As a matter of fact, the unstable point appears slightly ahead of the stable due to the asymptotic separation $g_{+}(J \rightarrow \infty)$ $-g_{-}(J \rightarrow \infty) \sim \mathcal{O}(\delta)>0$. As $\sigma_{0}$ grows, the $g_{ \pm}$curves move upward and the resonance moves toward $J=0$, and when $k_{0} \approx 2 \pi$ the unstable point undergoes an inverse tangency with the central point located at relatively smaller values of $J$. In terms of stability diagrams $\alpha$ vs $\sigma_{0}$, the mechanism is represented by the $\delta=2.0 \times 10^{-3}$ curve of Fig. 4 . In the diagram the onset of the resonance is observed as a direct tangent bifurcation with the creation of two matched solutions-one stable with $\alpha<1$ and one unstable with $\alpha$ $>1$. The bifurcation is indicated by the letter $a$ in Fig. 4(c) and occurs at $2 \sigma_{0}=2 \pi\left(\sigma_{0}=180^{\circ}\right)$ as expected.

As $\sigma_{0}$ keeps growing, the resonance migrates toward $r_{b 0}$, and $k\left(r_{b, \max }\right)$ approaches $k_{0}$. In Fig. 5(d) a Poincaré plot for $\delta=2.0 \times 10^{-3}$ and $\sigma_{0}=207^{\circ}$ shows the three fixed points corresponding to the original matched solution plus the just created stable and unstable matched solutions. By further increasing $\sigma_{0}$ one eventually meets the condition $k\left(r_{b, \max }\right)$ $=k_{0}$, where the original fixed point undergoes an inverse tangent bifurcation with the unstable fixed point of the resonance, in agreement with what was explained above. At this point, indicated by letter $b$ in Fig. 4(c), the original matched solution vanishes. However, one is still left with the stable matched solution of the resonance. As $\sigma_{0}$ increases further the sequence is reproduced: the new stable fixed point undergoes a direct followed by inverse period doubling bifurcations at $\alpha=-1\left[\sigma_{0} \approx 300^{\circ}\right.$ in Fig. 4(a) ], and an inverse tangent bifurcation at $\alpha=+1$ with an unstable fixed point of a yet newer resonance which invades the phase space when the new resonance condition $2 \sigma_{0}=4 \pi$ is met $\left[\sigma_{0} \approx 400^{\circ}\right.$ in Fig. 4(a)]. One is then left with a newer stable matched solution, now associated with the $k\left(r_{b, \max }\right)=4 \pi$ resonance. The whole process repeats itself roughly every $180^{\circ}$ in $\sigma_{0}$, for increasingly larger values of the positive integer $n$ indexing the general resonance condition $k\left(r_{b, \max }\right)=2 n \pi$. Therefore, excluding some small unstable bands where $\alpha<-1$, one may always find a stable matched solution for the envelope equation, which persists and can be used to confine intense particle beams to tighter radii even if one operates the beam much beyond $\sigma_{0}=180^{\circ}$.

For larger $\delta$ 's, the scenario described above presents some changes. If $\delta$ is large enough to create the gap mentioned earlier, but not large enough to preclude the presence of tangent bifurcations similarly to Fig. 3(c), one is left without stable matched solutions immediately after the tangency. Only later, after the gap is cleared as $\sigma_{0}$ increases, does the stable point of the resonance make its appearance. Within the gap, no closed orbit remains in the phase space. In terms of stability diagrams, for $\delta=1.0$ one sees from Fig. 4(a) that the point remains absent in wide regions along the $\sigma_{0}$ axis (around $\sigma_{0}=200^{\circ}$ and around $\sigma_{0}=400^{\circ}$ ). These gaps are to be avoided if the goal is beam confinement. Now, by further increasing $\sigma_{0}$, the system indeed retrieves its closed orbits [11] and the stable fixed point appears in the phase space, as in the points marked by the letter $a$ in Fig. 4(a) confinement becomes possible again then. The Poincare plot of Fig. 5(e) considers $\sigma_{0}=292^{\circ}$ to show how stable typically is the fixed point after its reappearance; no chaotic activity can be seen in the panel. Therefore, for large $\delta$ 's one alternates windows of stability in $\sigma_{0}$ where a stable matched solution exists, and forbidden gaps (the meaning of "forbidden" taken in the context of confinement), where the matched solution either exists but is unstable, or is simply absent. The purely monotonic case exemplified by Fig. 3(b) is similar to the previous one except for the fact that there is no tangent bifurcation. We shall not discuss this situation here.

\section{Obtaining tighter radii}

A figure of merit, if one is interested in beams with the smallest possible transverse dimensions, is the minimum value attained by the matched and stable beam envelope as it oscillates as a function of $s$. Analysis is thus in order of the minimum oscillatory value of $r_{b}$, let us call it $r_{b}^{*}$, versus the 


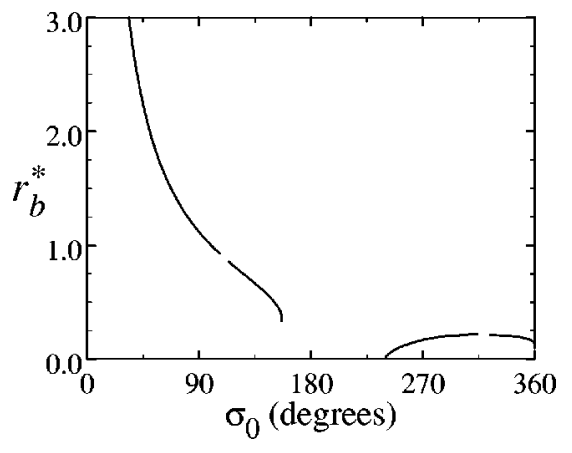

FIG. 6. The minimum oscillatory radius of stable matched solutions $r_{b}^{*}$ as a function of $\sigma_{0}$ for $K=3.0$ and $\delta=1.0$.

strength of the focusing magnetic field $\sigma_{0}$. The analysis is displayed in Fig. 6. We take $\delta=1.0$ and compare $r_{b}^{*}$ of the original fixed point with that of the stable fixed point of the $k\left(r_{b, \max }\right)=2 \pi$ resonance. The most useful ranges in both cases are the ones preceding the respective period doublings. Even though stability is recovered, the fixed points become closely surrounded by an increasing number of high-order resonances [such as in Fig. 5(c), where one sees the third and fourth order resonances] which may overlap leading to chaos [9]; we shall discuss the issue in more detail in the next section. It is evident from Fig. 6 that $r_{b}^{*}$ corresponding to $k\left(r_{b, \max }\right)=2 \pi$ is appreciably smaller than the one corresponding to the original fixed point. To perform a quantitative comparison, we take two points-one corresponding to the original fixed point and one to the resonance stable fixed point-both preceding the respective period doublings (small gaps in Fig. 6) and with the same $\alpha$ such that their stability characteristics are similar. We take here the points $\sigma_{0}=80^{\circ}$ and $\sigma_{0}=292^{\circ}$, both with $\alpha=-0.56$ [see Fig. 4(a)], the latter corresponding to the case displayed in Figs. 4(a)-4(c). As one moves from one point to the other, the magnetic field, which is proportional to $\sigma_{0}$, increases 3.65 times, whereas $r_{b}^{*}$ decreases 6.0 times, as seen in Fig. 3(a). In other words, the decrease in $r_{b}^{*}$ is almost twice the increase in $\sigma_{0}$ if one uses the resonance stable matched solution instead of the original matched solution. In other words, an increase of the magnetic field within its own order of magnitude produces a noticeable reduction in the minimum oscillatory radius of the stable matched solution. Presumably, $r_{b}^{*}$ becomes even smaller as one further increases the magnetic field and moves to stable fixed points of resonances $k\left(r_{b, \max }\right)=2 n \pi$ with larger values of $n$.

\section{Parameter-space analysis}

An important issue from the experimental point of view is to determine in the parameter space the regions of stability and of existence of the instability gaps. Therefore in this section we construct and discuss these parameter-space plots.

The plots are obtained by using the Newton-Raphson method to numerically determine the curves of $K$ as a function of $\sigma_{0}$ for which a bifurcation with $\alpha= \pm 1$ occurs, for a fixed $\delta$. The results are shown in Fig. 7, for $\delta=0.5$ [Fig. $7(\mathrm{a})]$ and $\delta=1.0$ [Fig. 7(b)]. The white regions in the figure correspond to parameter values for which at least one stable
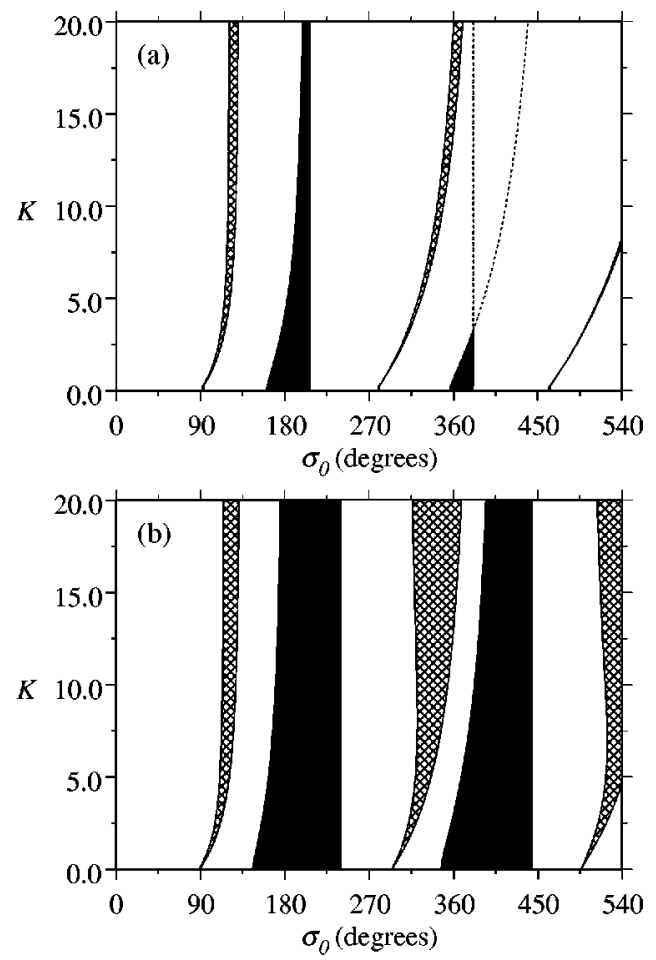

FIG. 7. Parameter-space plots obtained for (a) $\delta=0.5$ and (b) $\delta=1.0$. White regions correspond to parameter values for which at least one stable matched solution exists, shaded regions to the existence of a single matched solution which is unstable due to a period doubling bifurcation, and black regions to the gaps where no matched solution is found.

matched solution with $|\alpha|<1$ exists. The shaded regions correspond to the existence of a single matched solution with $\alpha<-1$, which is unstable because a period doubling bifurcation has taken place. The black regions correspond to the gaps where no matched solution is found. It is worth saying that, as will be discussed in detail in Sec. IV, the useful regions for beam transport are the stable (white) ones preceding the corresponding period doublings.

First of all, it is interesting to note that predictions of the model developed in Sec. II for the $n=1$ resonance can be extended to qualitatively understand what happens for higher-order resonances. In particular, the model predicts that for sufficiently small $\delta$ and large enough $K$ the instability gaps with no matched solutions (black regions) tend to disappear. That is seen in Fig. 7(a) for the $n=2$ resonance around $\sigma_{0}=360^{\circ}$. There, the gap is present for perveance lower than $K \approx 3.3$ because the inverse tangent bifurcation of the stable matched solution of the $k=2 \pi$ resonance with the unstable matched solution of the $k=4 \pi$ resonance occurs before the onset of the stable matched solution of the $k$ $=4 \pi$ resonance; i.e., the stable matched solution of a previous resonance bifurcates and disappears before (in terms of increasing magnetic field) the onset of the stable matched solution of the next resonance. According to the model, for such low perveance the process is rather linear, as in the case shown in Fig. 3(b), where the depression $g_{+}$is smaller than $g_{+}(J \rightarrow \infty)-g_{-}(J \rightarrow \infty)$, leading to the gap. As $K$ increases, nonlinearity also increases and the depression in $g_{+}$also 
increases. Above $K \approx 3.3$ the depression in $g_{+}$becomes larger than $g_{+}(J \rightarrow \infty)-g_{-}(J \rightarrow \infty)$, as in the case of Fig. 3(a), the gap disappears, and three matched solutions can be found. That is what happens in the parameter region limited by the dashed lines (which represent $\alpha=+1$ ) in Fig. 7(a).

Another interesting feature that can be understood from the model is that the onset of the additional stable matched solutions, which are the bifurcations leading to the lines that limit the black regions to the right, is essentially independent of the perveance $K$. These bifurcations occur when the $g_{-}$ curve shown in Fig. 3 crosses zero. Since $g_{-}$is a monotonically increasing function of $J$, the bifurcation always takes place at $J \rightarrow \infty$, meaning that the stable matched solution oscillates from $r_{b}=0$ to $r_{b} \rightarrow \infty$. Because in this case the particles of the beam spend most of the time far away from each other, space-charge effects introduced by $K$ are unimportant.

Regarding the overall beam transport stability, Fig. 7 shows that the perveance and the order of the resonance $n$ play a tricky role. In particular, the size of the instability gap caused by the period doubling bifurcation (shaded region) tends to increase with increasing $K$ and/or $n$. On the other hand, as discussed above for the perveance, the size of the gap where matched solutions are absent (black regions) tends to decrease as $K$ and/or $n$ increases. As for the amplitude of oscillations of the magnetic field, by comparing Figs. 7(a) and 7(b) it is clear that increasing $\delta$ tends to increase the size of all instability gaps.

\section{FULLY SELF-CONSISTENT SIMULATIONS}

We perform self-consistent simulations using up to $N$ $=2000$ macroparticles undergoing Coulomb interaction via static potentials generated by the Green's function method [12]. Particles are launched according to a KapchinskijVladimirskij (KV) distribution [1] but their finite number in the initial condition acts as the seed for any possible instability to develop. As the beam propagates along the focusing channel we compute the self-consistently obtained KV beam radius

$$
\bar{r} \equiv \sqrt{2\left\langle r^{2}\right\rangle}, \quad\langle Q\rangle \equiv \frac{1}{N} \sum_{j=1}^{N} Q_{j},
$$

which is $\sqrt{2}$ times the rms radius [9], and the (transverse) emittances

$$
\epsilon_{\zeta} \equiv 4 \sqrt{\left\langle\zeta^{2}\right\rangle\left\langle\zeta^{\prime 2}\right\rangle-\left\langle\zeta \zeta^{\prime}\right\rangle^{2}}, \quad \zeta \equiv x, y .
$$

Let us take $K=3.0, \delta=1.0$, and $\sigma_{0}=292^{\circ}$ to investigate one of the just found stable matched solutions as suggested by the envelope simulations pictured in Fig. 4. From the figure the envelope simulations tells us that at this point there is a stable matched solution and we want to check this information against this more general self-consistent particle simulation with Coulomb interaction. We first compare both radii $r_{b}$ and $\bar{r}$ in their final 10 periods, in a run of $s_{\max }=100$. This is done in Fig. 8(a), which indicates that the envelope model is extremely accurate and that in fact the stable matched solution persists within the context of the fully selfconsistent simulation. Figure 8(b), in its turn, shows that emittance is not increasing, which is a fact signaling that good beam quality is preserved.

In Fig. 8(c) we display the initial/final particle distribution over the $\left(X, P_{x}\right)$ cross section, and in Fig. $8(\mathrm{~d})$ we display the initial/final distribution over an $(X, Y)$ cross section, $X$ $\equiv x / \bar{r}, P_{x} \equiv \bar{r} p_{x}-x(d \bar{r} / d s), Y \equiv y / \bar{r}$ [9]. These panels show again that the beam does not undergo macroscopic changes along the focusing channel. The small dispersion is due to discrete particle effects and it is reduced as more and more particles are used, but the important feature to be noticed here is that the bulk of the particle distribution remains confined within the limits $(-1,+1)$ along all the normalized axes $X, Y$, and $P_{x}$, even after this long computer run.

Now consider a point right inside one of the gaps, such as for instance, the one with the same values of $K$ and $\delta$ as above, but with $\sigma_{0}=203^{\circ}$, in Fig. 9; we refer the reader once more to Fig. 4. Self-consistent simulations and beam envelope simulations are in total agreement again, as indicated by Fig. 9(a). However, this very same panel shows that the mean radius increases within a short period of time-note that this run is only up to $s_{\max }=3$. The unbounded radius growth is a direct result of the fact that within the gaps there is no trapping fixed point which would be able to generate closed orbits and the resulting limits on the oscillations of $\bar{r}$ or $r_{b}$. The initial/final cross sections now reveal the following: a noticeable distortion in the $\left(X, P_{x}\right)$ space as seen in Fig. 9(d), which is in fact related to the $\epsilon_{x}$ growth as shown in Fig. 9(b). For a short run like this the beam preserves the circular shape of Fig. 9(c).

The final analysis of this section is devoted to the matched solution when it comes back from its own period doubling, such as, for instance, the point $\sigma_{0}=135^{\circ}$ of Fig. 10, again with $K=3.0, \delta=1.0$, and $s_{\max }=100$. The results are quite similar to the ones displayed in Fig. 8, with the noticeable exception of Fig. 10(b) where it becomes clear that a steady emittance growth is present, as opposed to the corresponding Fig. 8(b). There is a belief that emittance growth under such circumstances can be caused by the nearby presence of higher-order resonances, as seen in Fig. 5(c), which can act upon discrete aspects of particle distributions [9]. As mentioned earlier, if one were working with a genuinely continuous and homogeneous KV distribution, emittance would be constant since in this case the envelope equation and the associated constant emittance become exact results.

All in all, and considering a series of similar analyses not shown here, we can state that the matched solutions created here can be used to confine beams as long as one operates before the corresponding period doubling. Afterward, the stability curve becomes too steep, as can be observed in Fig. 4, and this allows for a close packing of higher-order resonances in general, as seen in Fig. 5(c).

\section{FINAL CONCLUSIONS}

To summarize, in the present paper we extend previous work [10] and perform a stability analysis of periodically focused intense particle beams based on analytical estimates, 

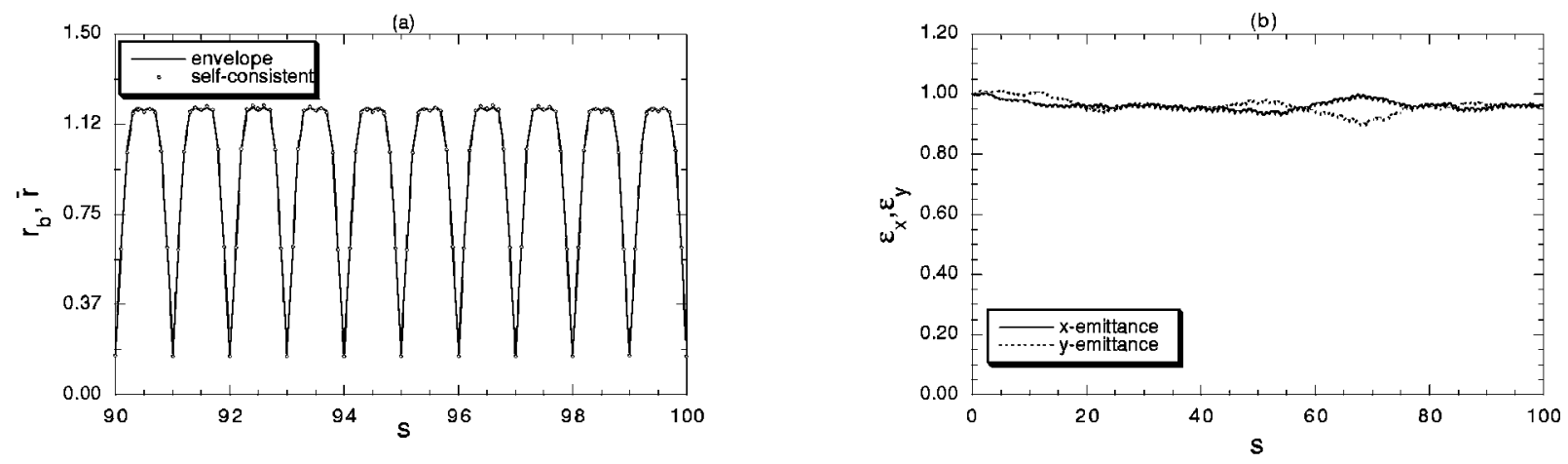

(c)
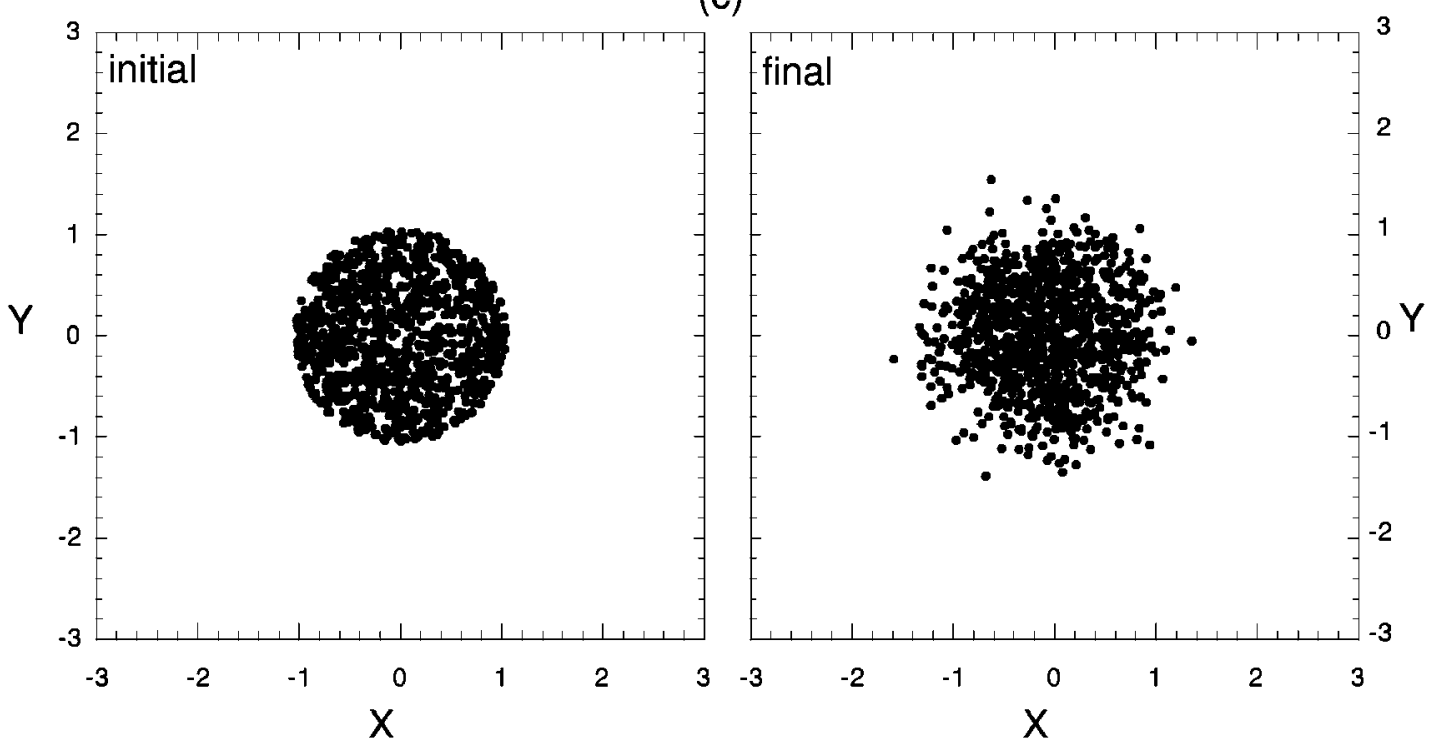

(d)
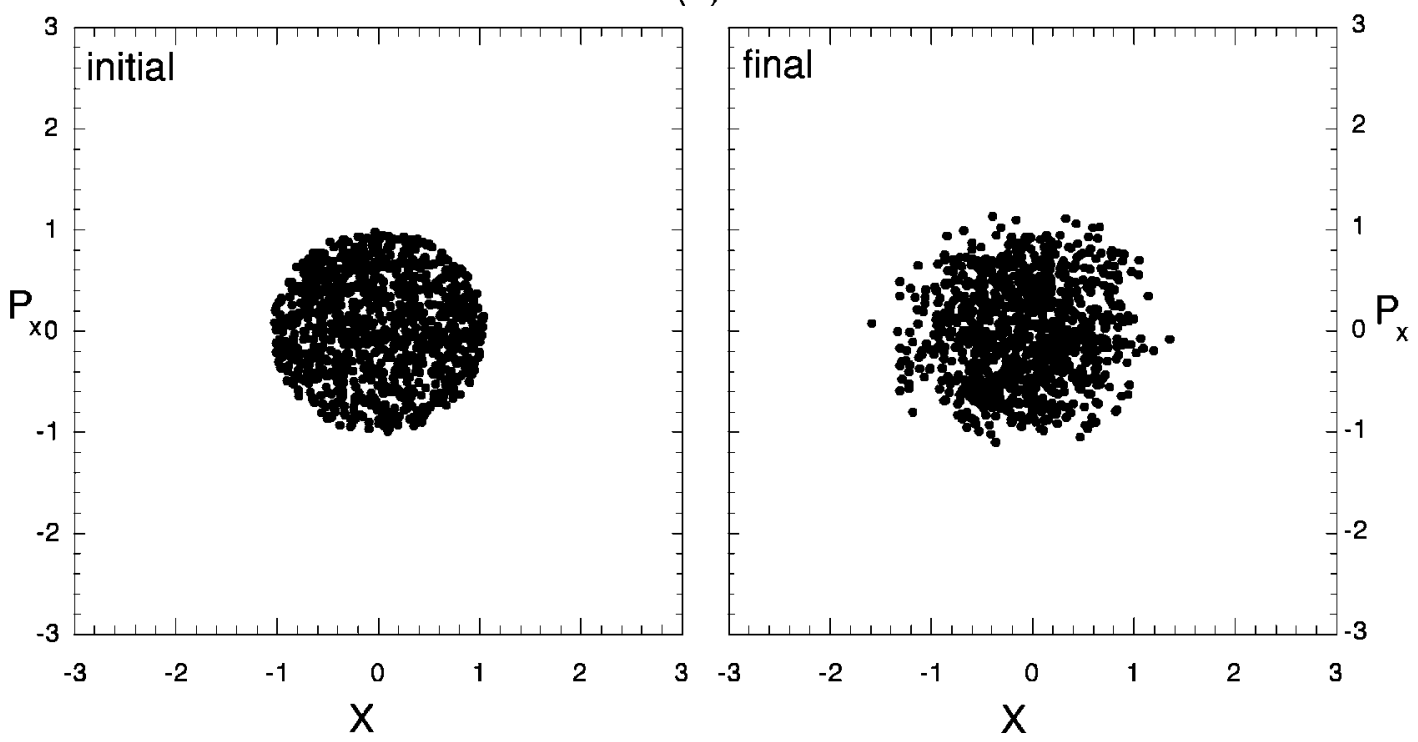

FIG. 8. Self-consistent simulations for a matched solution with $K=3.0, \delta=1.0, \sigma_{0}=292^{\circ}$, and $s_{\max }=100$. In (a) $\bar{r}$ and $r_{b}$ versus $s$; in (b) $\varepsilon_{x}$ and $\varepsilon_{y}$ versus $s$; in (c) initial and final cross sections $(X, Y)$; and in (d) initial and final cross sections $\left(X, P_{x}\right) . r_{b}(s=0)=\bar{r}(s=0)$ $=0.1673, p_{b}(s=0)=d \bar{r} / d s(s=0)=0$. 

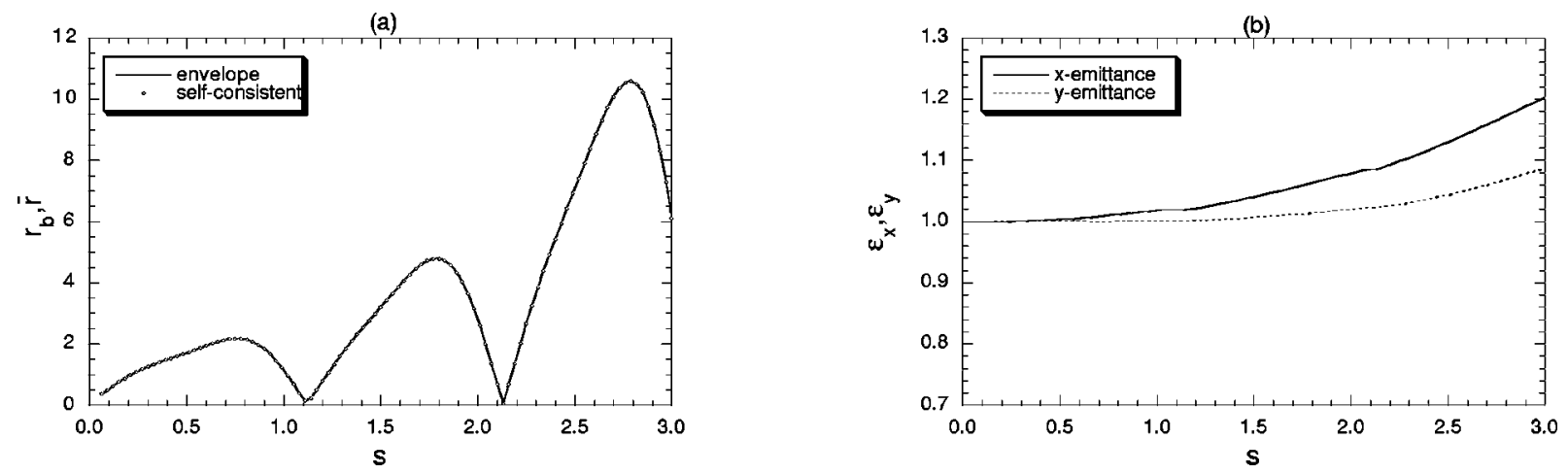

(c)
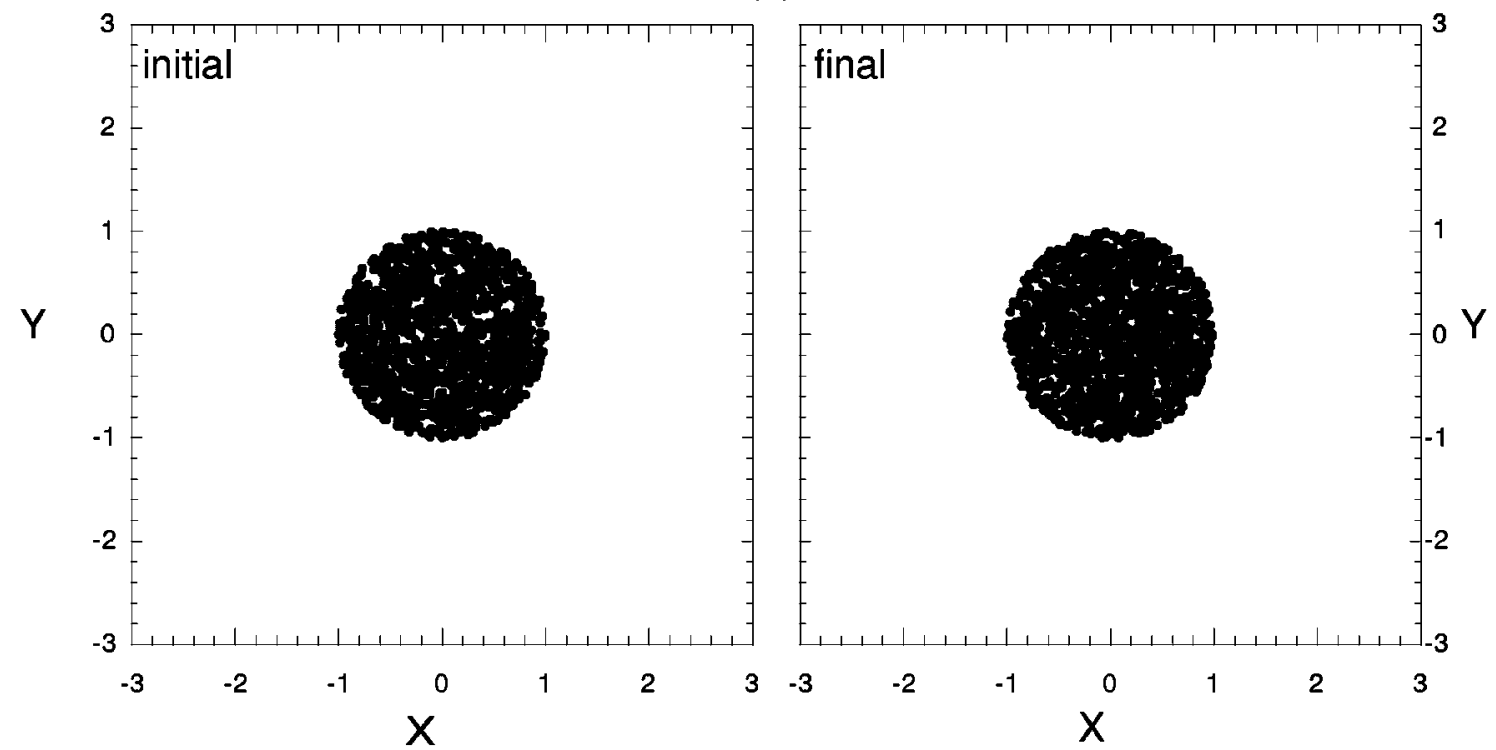

(d)
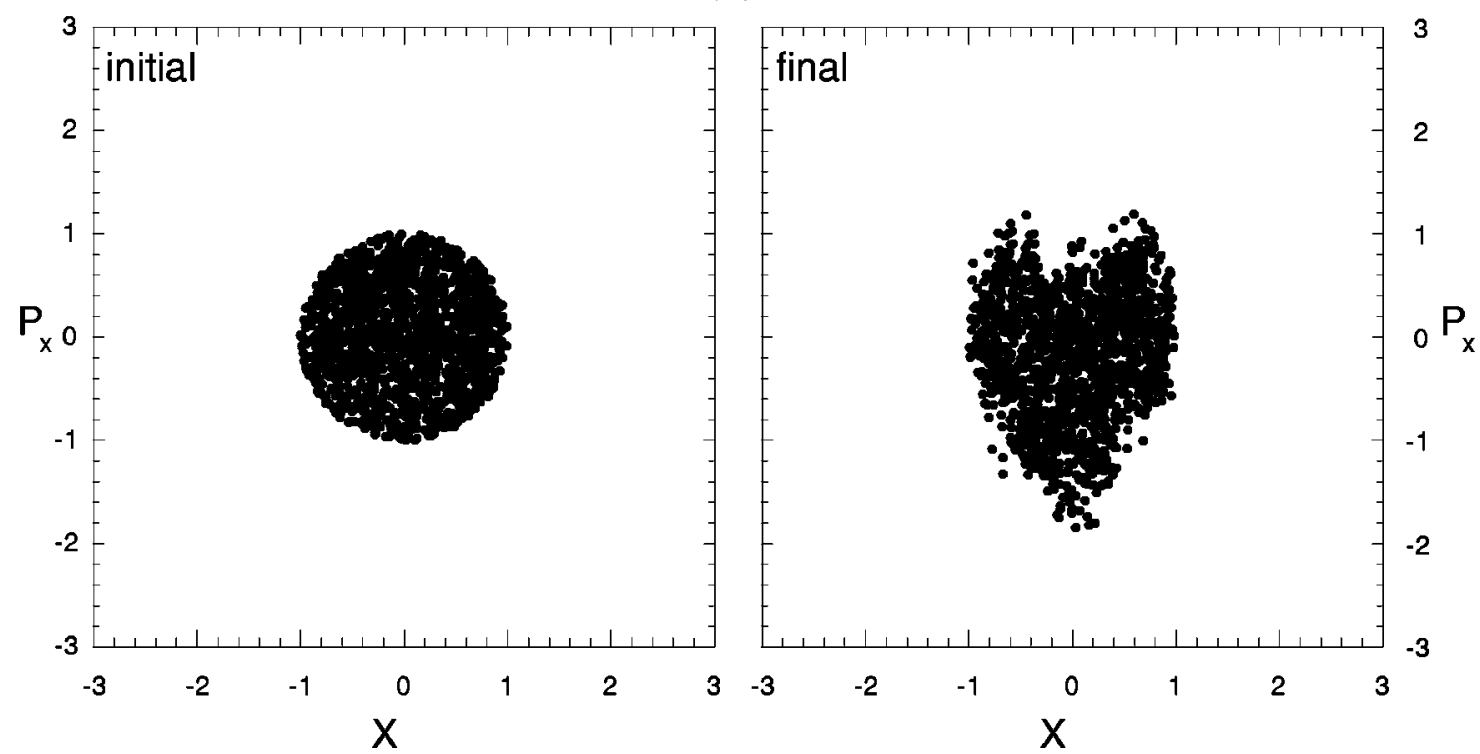

FIG. 9. The same kind of panels as in Fig. 8, but now within a gap. Parameters and initial conditions are the same except $\sigma_{0}=203^{\circ}$ and $s_{\text {max }}=3.0$ now. 

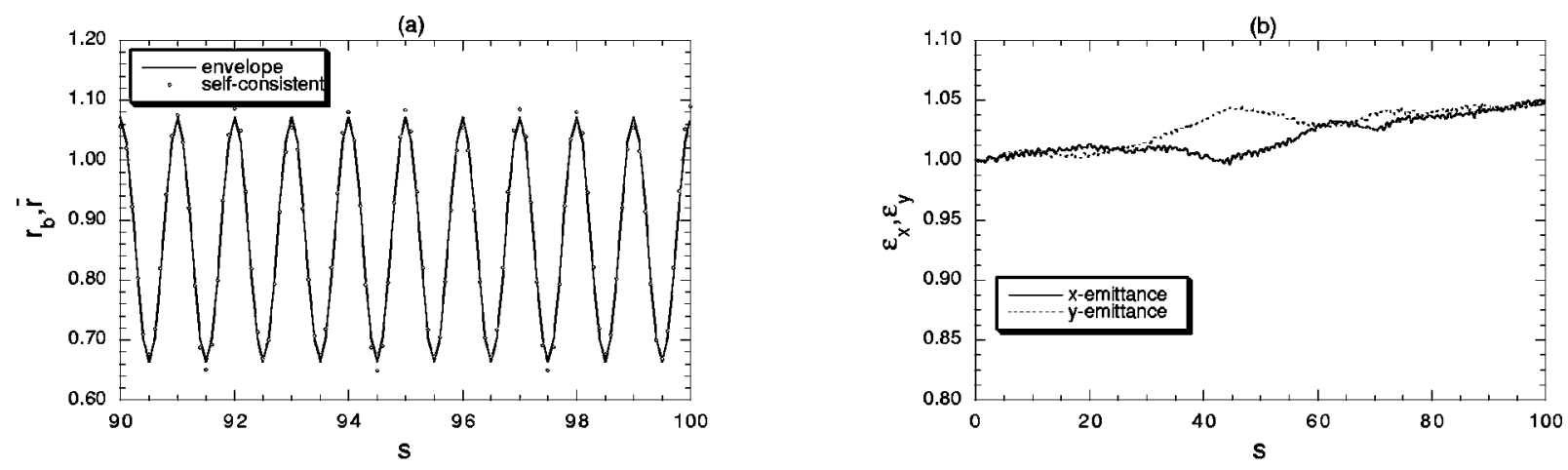

(c)
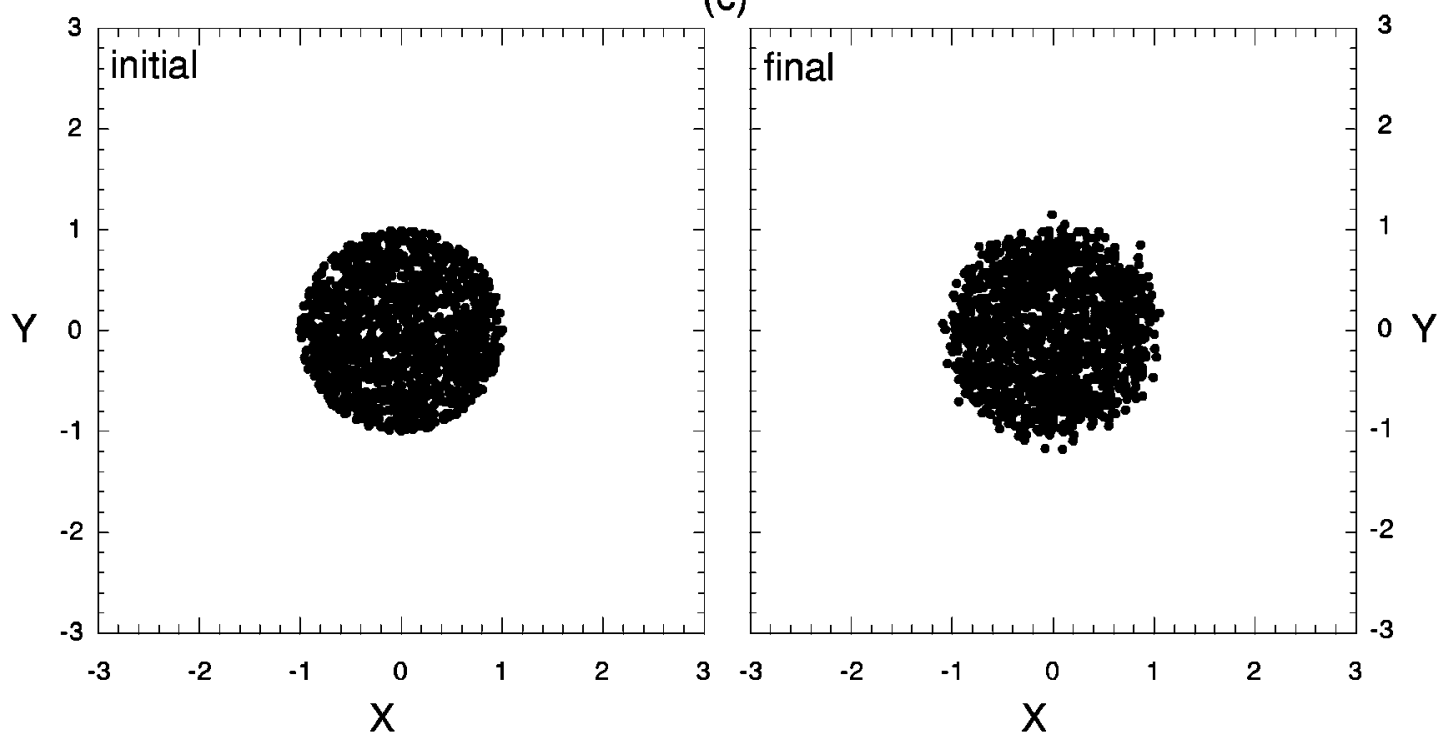

(d)
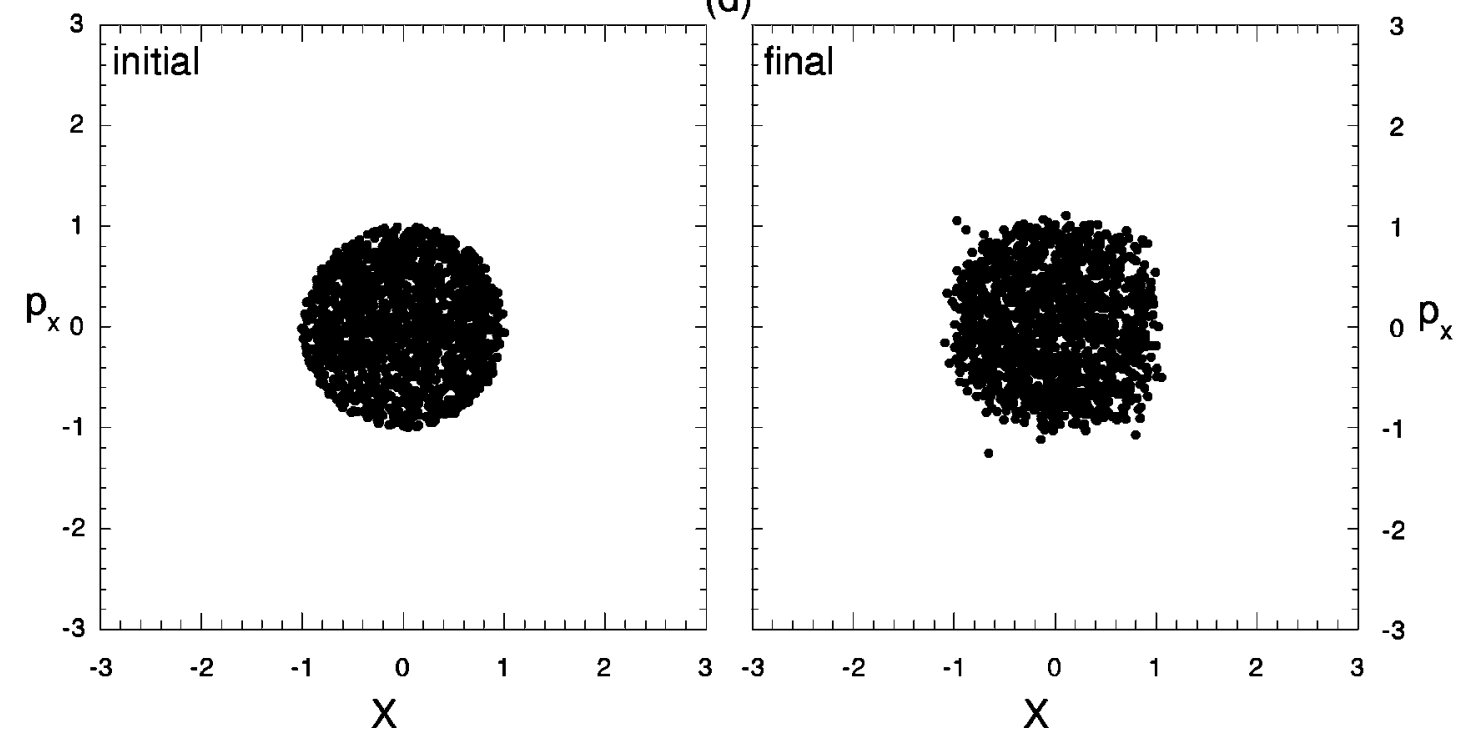

FIG. 10. The same kind of panels as in Fig. 8, but now right after an inverse period doubling. Parameters and initial conditions are the same except that $\sigma_{0}=135^{\circ}$ and $r_{b}(s=0)=\bar{r}(s=0)=1.157$ now.

on the beam envelope equation, and on fully self-consistent simulations. An analytical model is developed in order to understand the overall nonlinear dynamics of the system. One particular result from the model is the interplay between the various defocusing forces on beam dynamics. Dense beams dominated by space-charge effects exhibit nonlinear features, like tangent bifurcations, which are not observed in the absence of space-charge effects, when the beam becomes 
dominated by thermal and rigid rotation effects, with the dynamics becoming linear. Gaps may be formed as we vary the focusing strength, within which no matched solution can be found. This makes the issue of critical relevance if the goal is to operate with stable periodic solutions. We show that matched solutions undergo a series of direct and inverse bifurcations as the parameters of the system are varied, and that, although the original matched solution analyzed in previous works becomes unstable and eventually vanishes as the focusing field strength increases, stability is recovered for yet larger fields.

Then the Poincare mapping technique allied to the Newton-Raphson method [11] is employed to precisely locate and determine the existence and stability of matched solutions in the phase space of the beam envelope model. All the results based on analytical calculations and approximations are confirmed here. We also show how can one effectively focus beams to tighter radii using the stable matched solutions found for focusing strengths greater than the previously established threshold.

The results are finally validated with self-consistent simulations. The general conclusion is that the beam model is good enough apart from emittance growth detected only in the full simulations. Emittance growth is small in the case of matched solutions, but can become relatively large within the gaps.

In our investigation $\kappa_{z}(s)$ is a harmonic function of the distance $s$, but we also found that bifurcations for other forms of $\kappa_{z}(s)$ have the same qualitative behavior as long as the periodicity condition $\kappa_{z}(s+1)=\kappa_{z}(s)$ holds. In addition, our investigation of stability zones in the $K$ vs $\sigma_{0}$ parameter space reveals that even for much larger values of $K$, such as $K=100$, these stability zones are still present.

What we see from the overall investigation is that the scenario as one increases the focusing field is not the existence of a single threshold above which confinement is impossible, as believed so far, but the existence of regions of stability interrupted by gaps where the matched solution either becomes unstable or is completely absent.

As a very final comment we note that the approximation of constant longitudinal momentum is used throughout this paper. It has been shown in general terms that the approximation is accurate in the case of paraxial beams with nonrelativistic transverse motion [9] investigated here. Furthermore, in our specific case the additional degree of freedom associated with small longitudinal corrections would have an equally small effect on the very stable and robust type of matched phase space detected here-see Fig. 5(e) where one observes enhanced stability arising from the complete absence of resonant islands around the matched solution. If one wishes to describe end effects associated with short particle bunches, corrections to the paraxial approximation will be needed.

\section{ACKNOWLEDGMENTS}

We acknowledge support from CNPq, Brazil. Numerical simulations where partially performed with the Cray Supercomputer of Universidade Federal do Rio Grande do Sul.

\section{APPENDIX}

Suppose one has an action-angle represented Hamiltonian in the form of Eq. (13):

$$
H_{0}=k_{0} J+\alpha J^{3 / 2} \sin ^{3} \theta+\beta J^{2} \sin ^{4} \theta .
$$

Suppose $J \ll 1$ and define a generating function $F(\mathcal{J}, \theta)$ with which to get rid of the $\theta$ dependence above. Suppose also that $F(\mathcal{J}, \theta)=\theta \mathcal{J}+F_{1}+F_{2}+\cdots, F_{n} \sim O\left(\mathcal{J}^{(n+2) / 2}\right)$. Then we write

$$
H_{0}\left(\mathcal{J}+\frac{\partial F}{\partial \theta}, \theta\right)=h(\mathcal{J})
$$

To first order

$$
\begin{aligned}
& k_{0} \frac{\partial F_{1}}{\partial \theta}+\alpha \mathcal{J}^{3 / 2} \sin ^{3} \theta=0 \\
& \quad \Rightarrow h(\mathcal{J}) \\
& \quad=k_{0} \mathcal{J}+O\left(\mathcal{J}^{2}\right) .
\end{aligned}
$$

To second order

$$
\begin{aligned}
& k_{0} \frac{\partial F_{2}}{\partial \theta}+\left[\frac{3 \alpha}{2} \frac{\partial F_{1}}{\partial \theta} \mathcal{J}^{1 / 2} \sin ^{3} \theta+\beta \mathcal{J}^{2} \sin ^{4} \theta\right]_{f}=0 \\
& \quad \Rightarrow h(\mathcal{J}) \\
& \quad=k_{0} \mathcal{J}+\mu \mathcal{J}^{2},
\end{aligned}
$$

with

$$
\mu=\frac{3 \beta}{8}-\frac{15 \alpha^{2}}{32 k_{0}}
$$

as in Eq. (14), and where the subscript $f$ denotes the fluctuational part of the bracketed expression.
[1] I.M. Kapchinskij and V.V. Vladimirskij, in Proceedings of the International Conference on High Energy Accelerators (CERN, Geneva, 1959), p. 274.

[2] I. Hofmann, L.J. Laslett, L. Smith, and I. Haber, Part. Accel. 13, 145 (1983).
[3] R.C. Davidson, Physics of Nonneutral Plasmas (AddisonWesley, Reading, MA, 1990).

[4] M. Reiser, Theory and Design of Charged-Particle Beams (Wiley and Sons, New York, 1994).

[5] C. Chen, R. Pakter, and R.C. Davidson, Phys. Rev. Lett. 79, 
225 (1997).

[6] See, e.g., Space Charge Dominated Beams and Applications of High-Brightness Beams, edited by S.Y. Lee, AIP Conf. Proc. No. 377 (AIP, Woodbury, NY, 1996).

[7] P.M. Lapostolle, IEEE Trans. Nucl. Sci. NS-18, 1101 (1971); F.J. Sacherer, ibid. NS-18, 1105 (1971).

[8] C.J. Struckmeier and M. Reiser, Part. Accel. 14, 227 (1984).

[9] C. Chen and R.C. Davidson, Phys. Rev. Lett., 72, 2195 (1994); Phys. Rev. E 49, 5679 (1994).
[10] R. Pakter and F.B. Rizzato, Phys. Rev. Lett. 87, 044801 (2001).

[11] A.J. Lichtenberg and M.A. Lieberman, Regular and Stochastic Motion (Springer-Verlag, New York, 1992); R. Pakter, G. Corso, T.S. Caetano, D. Dillenburg, and F.B. Rizzato, Phys. Plasmas 1, 4099 (1994); J. Guckenheimer and P. Holmes, Nonlinear Oscillations, Dynamical Systems, and Bifurcations of Vector Fields (Springer-Verlag, New York, 1990).

[12] R. Pakter and C. Chen, Phys. Rev. E 62, 2789 (2000). 